\title{
Ralph Christensen Postmoderne Methodik oder: Überlebt der König seine Enthauptung in der Regel?
}

Zu schildern ist die Abdankung der Monarchie und der allmähliche Übergang zu demokratischen Strukturen im Gebiet juristischer Methodik. Anlass ist ein Buch von Ino Augsberg. ${ }^{1}$ Darin wird die Lesbarkeit des Rechts in acht texttheoretischen Lektionen untersucht. Zunächst stellt der Autor die vordemokratischen Voraussetzungen der herkömmlichen Methodik dar, um aus der Kritik die Grundlagen einer demokratischen Methodenlehre zu gewinnen. Was heißt also demokratische Rückbindung für eine richterliche Entscheidung?

\section{Die Enthauptung des Königs}

Die Verknüpfung von Demokratie und richterlichem Urteil liegt für die herkömmliche Theorie darin, dass der Richter den Inhalt der Gesetze auf den Fall anwendet. Die Vermittlungsinstanz ist die Sprache, welche dem Richter die objektive Bedeutung des Textes vorgibt. Unter allen Arten, den Text zu lesen, gibt es immer eine höchste und beste: die wörtliche Bedeutung. Sie ist die sichere Grundlage richterlicher Erkenntnis. Aber sagt Augsberg: „Keine Lektüre kann buchstäblich genommen werden."2 Das scheinbar stabile Gebäude der Monarchie hat also massive Probleme im Fundament. Sie werden sichtbar, wenn man den Ausweis der wörtlichen Bedeutung fordert: Schon beginnt ein erbitterter Streit, ob man sie beim Autor, beim Text oder beim Leser findet. Einig ist man sich nur, dass es in dieser Verbindung von drei Elementen ein dominierendes Subjekt gibt, welches die beiden anderen auf den Status passiver Objekte reduziert. Augsberg inspiziert die Provinzfürsten, von denen jeder König sein will, nacheinander.

\section{a) Institutionenpositivismus oder Der Tod des Autors}

In dem Film „Stadtneurotiker“ lässt Woody Allen in einem Streit um die Interpretation der Medientheorie Marshall McLuhans diesen selbst erscheinen, um die gegnerische Interpretation zu widerlegen. Augsberg zitiert den die Episode abschließenden Satz: Wenn das Leben nur so wäre. ${ }^{3}$ Dann könnte man bei Interpretationsstreitigkeiten immer den Autor fragen oder mindestens die subjektive Auslegungstheorie als ihren Stellvertreter. Aber auch der Autor weiß es nicht. Denn er hat sich wie McLuhan den Medien überantwortet und kann nicht mehr überblicken, was er sagt. Juristen können dies auch bei Radbruch ${ }^{4}$ nachlesen, der sich wiederum auf Goethe beruft mit dem Diktum, kein Weber wisse, was er webt. Auch der Autor kann danach nur als der ewige Besitzer seines Werkes funktionieren, wenn er es nicht aus der Hand gibt. Sobald er dies aber tut, ver-

1 Ino Augsberg, Die Lesbarkeit des Rechts, Weilerswist 2009. Seitenangaben beziehen sich im Folgenden darauf.

2 S. 30.

3 Augsberg (Fn. 1), S. 95.

4 Vgl. Gustav Radbruch, Rechtsphilosophie, 1973, S. 212 f. 
schwindet seine Deutungshoheit im Kommentar und der Vielzahl der Stimmen seiner Leser.

\section{b) Gesetzespositivismus oder Das Schweigen der Gesetze}

Aber es gibt neben dem Autor als Disziplinierungsinstrument für die Leser noch den Kommentar. ${ }^{5}$ Danach ist der Leser darauf beschränkt, zum ersten Mal auszusprechen, was im Text schon immer gesagt war. Dieses Prinzip ist Juristen viel plausibler. Denn bei jeder praktischen Entscheidung geht der Richter von den Stichworten in der Akte aus, um über das Gesetz in den Kommentar zu gelangen. Beim Amtsgericht ist der Palandt das Buch der Bücher, während die Obergerichte zwei oder sogar drei Kommentare lesen, um eine Meinung mehr zu kennen. Aber schon in der Mehrzahl der Kommentare zeigt sich ein Bruch. Außerdem weiß jeder Richter, dass er an kritischen Punkten die im Kommentar zitierten Vorentscheidungen selbst nachlesen muss. ${ }^{6}$ Wenn diese dann nicht passen oder sogar wie meistens widersprüchlich sind, muss er selbst in die Auslegung eintreten oder sogar mit Kollegen beziehungsweise Prozessbeteiligten diskutieren. Man sieht daran, dass ein Text keine allgemeine Grammatik aufweist, in welche der Leser mit seiner Kompetenz nur einrücken müsste. ${ }^{7}$ Wären die Gesetze nur auszusprechen, dann bräuchte man kein Verfahren. ${ }^{8}$ Auch die Hierarchisierung von Texten garantiert keine Entscheidung.

\section{c) Richterpositivismus oder Die Vielzabl der Stimmen}

Als letzter Halt kommt der Leser in Betracht. Wenn die Absicht des Gesetzgebers und das Prinzip der Kommentierung nicht ausreichen, um dem Richter die Entscheidung des Falles vorzugeben, vielleicht sind es die Stimmen der Kollegen, die ihn zurückholen aus der Nacht der Entscheidung in das Licht des Gesetzes. ${ }^{9}$ „Mit der Mündlichkeit verbindet sich ein Versprechen von absoluter Präsenz." ${ }^{10}$ Bei Husserl ja, ${ }^{11}$ im Alltag nein, möchte man sagen. Die Personalisierung des Textes ist entgegen Augsberg keine Kompensationsstrategie, sondern eine Überhöhungsstrategie. Präsenzmetaphysik hat außer in Sonntagsreden nie funktioniert. Dekonstruktion muss man deswegen nicht von außen an die Praxis herantragen, sondern man muss nur genau hinschauen. Schon der frühe Carl Schmitt wusste in „Gesetz und Urteil“, dass dies nicht funktioniert. Denn er weigerte sich, die Orientierung am anderen Richter als Zielpunkt jeder Auslegung ${ }^{12}$ entweder normativ oder empirisch zu begreifen. Damit war der andere Richter aber von vornherein eine Leerformel, der nur in der späteren Art- oder Rassegleichheit scheinbar mit Inhalt aufgeführt wurde. Tatsächlich ist die Kompetenz von Richtern nicht so homogen, wie diese Formel voraussetzt. Denn sonst hätte Carl Schmitt die Artgleichheit nicht bemühen müssen. Vor allem aber ist der Richter als Leser nicht allein in seiner Studierstube. Er ist eingebunden in ein Verfahren und einen Rechtszug. Dort muss er sich mit Argumenten gegen andere Lesarten behaupten. Zwar könnte er entscheiden, aber dann müsste er die Last der Begründung tragen,

Michel Foucault, Die Ordnung des Diskurses, Frankfurt am Main/Berlin/Wien 1977, S. 18. Das Prinzip der Kommentierung bedeutet hier, eine Hierarchie in Texte einzuziehen.

6 Vgl. zur Rolle juristischer Kommentare als Mittel der Hierarchisierung Peter Stegmaier, Wissen, was recht ist, Wiesbaden 2009, S. $210 \mathrm{ff}$.

7 Augsberg (Fn. 1), S. 33 f., 105, Fn. 38.

8 S. $60,62,77 \mathrm{f}$.

9 Diese Position unterstellt Augsberg (Fn. 1, S. 101) zu Unrecht der Strukturierenden Rechtslehre.

10 S. 85.

11 Vgl. dazu Jacques Derrida, Die Stimme und das Phänomen, Frankfurt am Main 1979, S. 51.

12 Augsberg, S. $72 \mathrm{f}$. 
dort alle vorgetragenen Argumente verarbeiten und eventuell mit Rechtsmitteln

rechnen. So wird das große Pathos der Entscheidung aufgelöst in viele kleine Anschlusszwänge.

\section{Das juristische Wissen nach dem Tod des Königs}

„Kopf ab“ resümiert Augsberg seine Durchsicht des juristischen Wissens über Auslegung und Texte. ${ }^{13} \mathrm{Im}$ Verbund von Autor, Text und Leser hat sich keine Instanz als herrschendes Subjekt erwiesen, welche die letzte und höchste Lesart garantieren könnte. Der Gesetzestext ist aber auch kein Puzzle, dessen Einheit durch eine Addition von Autorenabsicht, Kommentierung und Leserkompetenz hergestellt würde. Der Text existiert nur in einer streitigen Vielzahl von Lesarten. Die Entscheidung, welche dann die beste ist, geschieht nicht in einem friedlichen Netzwerk gleichberechtigter Elemente. Vielmehr bildet die Unterscheidung von Recht und Unrecht eher ein Rhizom, durchsetzt von umkämpften Hierarchien und bedroht durch hegemoniale Diskurse, welche der Logik des Baumes folgen. ${ }^{14}$ Auch die symmetrische Aufteilung der Kompetenzen in ein Netzwerk erweist sich als illusorisch. Der Text hat keine Einheit. Es stellt sich damit die Frage, ob der Faden zwischen Demokratie und gerichtlicher Entscheidung gerissen ist.

\section{a) Die juristische Methodik nach dem Positivismus}

Mit der Dekonstruktion verschwindet ein Begriff von Lesen, der dieses auf eine gegenwärtig vorgegebene und beherrschbare Bedeutung bezieht. Das Lesen wollte nur nachvollziehen, was schon da war. Aber die objektive oder wörtliche Bedeutung, über die man nicht streiten kann, gibt es nicht. Nur Fundamentalisten brauchen sie wirklich. Sie lassen zum Verständnis eines Textes ausschließlich ihre eigenen Kontexte gelten. Eine Religion mit heiligen Texten und wörtlicher Bedeutung lässt in hermeneutischen Kriegen andere dran glauben. Aber zum Glück ist das Recht keine solche Religion. Seine Sprache wird immer neu kontextualisierbar, und niemand kann beanspruchen, den Kontext erschöpfend zu beschreiben. Ohne diese wörtliche Bedeutung liefert uns die Sprache eines Textes kein objektives oder absolutes Wissen mehr, sondern nur noch mehr oder weniger gute Argumente. Eine Steuerung des Urteils durch die Sprache des Gesetzes ist damit nicht abgeschafft, sie ist aber verwiesen auf Argumentation.

Wenn die Sprache keine Objektivität garantieren kann, scheitert ein theologisches Modell der Gesetzesbindung, welches das Sprechen des Richters aus einer höheren Instanz legitimiert. Der Richter muss nicht mehr als Bauchredner des Gesetzes auftreten. Er steht jetzt unter dem hermeneutischen Imperativ ${ }^{15} \mathrm{zu}$ verstehen, aber sein Verständnis nicht als endgültig und objektiv aufzufassen, sondern als revisibel. Aber trotzdem will Augsberg die Gesetzesbindung als Rahmen richterlichen Tuns beibehalten und fordert für ihre Überprüfung eine robuste Methodik. Wie soll diese Bindung funktionieren?

\section{b) Das Gesetz nach dem Positivismus}

Die Interpretation des Gesetzes läuft jetzt nicht mehr über Regeln, sondern über Daumenregeln. Muddling Through statt Erkenntnis. ${ }^{16}$ Damit wird reflektiert,

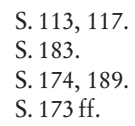


dass methodische Regeln keine fraglose Objektivität beanspruchen können ${ }^{17}$ und vor allem als implizites Wissen der Praxis anzusehen sind. ${ }^{18}$ Trotzdem soll der Richter aus dem Text des Gesetzes eine motivierte und voraussagbare Interpretation ableiten können. ${ }^{19}$ Woraus soll sich diese Voraussagbarkeit ergeben?

Unser Autor kennt zum Glück den höchsten Zweck des Rechts, welcher darin besteht, Erwartungsstabilität zu garantieren. ${ }^{20}$ Auf diesen höchsten Zweck kann man sich mit Hilfe der teleologischen Auslegung beziehen, die man dann noch bereichsspezifisch konkretisieren kann. ${ }^{21}$ Außerdem hilft der Bezug auf Sozialkonventionen, wobei deren Aufnahme allerdings auch Dissens ermöglichen muss. ${ }^{22}$ Eine sehr voraussehbare Lesart für diesen Vorschlag wäre wohl die folgende: Der Autor kommt nach einer radikalen Kritik der herkömmlichen Auslegungslehre zum weithin akzeptierten Ergebnis, dass es für die Rechtsanwendung eine solide Grundlage im höchsten Zweck des Rechts gibt und damit die Teleologie die Krone der Auslegung ist. Auch Larenz und alle seine Wertungsjuristen können sich darüber freuen. Aber obwohl diese Lesart die voraussagbarste ist, kann sie entgegen dem eigenen Kriterium von Augsberg nicht als die beste angesehen werden. Er will mehr als eine knappe Verbeugung vor Ungewissheit mit anschließendem Übergang zum Immer-Schon. Er will die Unterscheidung von Recht und Unrecht nicht einfach den Richtern überantworten, sondern für demokratische Revisionen offenhalten. Deswegen braucht er weitere Unterscheidungen, die erst im Verfahren zum Tragen kommen.

\section{c) Das Verfabren nach dem Positivismus}

Juristische Entscheidungen werden von Experten mit Hilfe ihres Sonderwissens getroffen. Hat dieses juristische Wissen etwas mit demokratischen Vorgaben zu tun, oder ist es ihnen entzogen beziehungsweise vorgeordnet?

Die erste Rückbindung besteht zunächst darin, dass Richter ihr Urteil über die Begründung mit dem demokratisch zustande gekommenen Gesetz verknüpfen müssen. Um die Legitimität des Gesetzes auf das Urteil zu übertragen, müssen sich die tragenden Leitsätze als Lesart des Gesetzes behaupten können. Aus der neueren Textwissenschaft haben wir erfahren, dass keine Lesart mit einem Text notwendig verknüpft ist. Es gibt kein absolutes, das heißt von Kontexten losgelöstes Wissen über die Bedeutung eines Textes. Alle Voraussetzungen, die wir verwenden, sind vielmehr relativ zu unserer Auslegungskultur. Trotzdem benötigen wir Wissen, weil wir nicht kryptopolitisch urteilen wollen. ${ }^{23}$ Augsberg unterscheidet im juristischen Bereich stabileres Wissen von weniger stabilem Wissen. Das erste besteht in bereits anerkannten Auslegungsergebnissen (Präjudizien), das zweite in der Bearbeitung neuer Probleme (originäre Auslegung). In der heutigen Wissenschaftstheorie unterscheidet man epistemisches Wissen, welches praktisch bewährt ist, von thetischem Wissen, dem diese Bewährung noch

\footnotetext{
S. 174.

S. 175.

S. 177 .

S. $178 \mathrm{f}$.

S. 184.

S. $181 \mathrm{f}$.

Die große Entscheidung aus dem normativen Nichts ist auch für Augsberg keine Alternative. Genau wie die Rechtsordnung selbst will er diese undifferenzierte Größe in viele kleine Entscheidungen auflösen. Im Übrigen ist Carl Schmitt natürlich auf die Rolle des bad guy besetzt. Das ist wie mit Mario Adorf in den Karl-May-Filmen der 60er Jahre. Zwar hätte das deutsche Staatsrecht noch einige andere großen Schurken zu bieten, aber ihre Sprache ist eben wesentlich plumper als die von Carl Schmitt. Er dient bei Augsberg als Stichwortgeber für das Problem der Autonomie der Rechtsordnung. Dieser wichtige Argumentationsstrang wird hier aus Platzgründen unterdrückt.
} 
fehlt. ${ }^{24}$ Beide Formen von Wissen können in der freien Diskussion überprüft werden und gewinnen so eine bessere Rationalitätsgrundlage. Das epistemische Wissen ist durch seine Bewährung in der Praxis einfach schwerer zu erschüttern. Wer vom stabilen Wissen abweichen will, trägt dafür nach Augsberg eine Argumentationslast. Mit dem Stichwort Argumentation sind wir beim Verfahren und damit bei der Performanz beziehungsweise Performance des Rechts. Auch dies ist ein wichtiges Stichwort bei Augsberg, und es weist eine spezifische Ambivalenz auf.

Das Verfahren kann einfach der Trichter sein, ${ }^{25}$ mit dessen Hilfe dem Betroffenen sein Recht durch Lernen beigebogen wird. Wir haben dann Performanz als vorher schon feststehende Anwendung von Regeln. Alles, was darüber hinaus geht, ist lediglich Fehler. Damit gehört das Verfahren zur hierarchischen Logik des Baumes. So argumentiert Luhmann vor seiner Dekonstruktion. Nach seiner Auseinandersetzung mit Derrida sieht Luhmann ein, dass das Recht nicht einfach Probleme mit Erwartungssicherheit löst, sondern dass es sich dabei selbst verschiebt. Jetzt ist das Verfahren nicht einfach die kompetente Durchführung von Regeln, sondern es kann sich darin Neues ereignen als Performance. Das Verfahren wird damit zum Teil eines Rhizoms. Mit jeder Anwendung einer Regel wird sie in eine neue Umgebung gestellt und verschiebt so ihren Sinn. So schreibt sie sich in der Iteration fort und ist nicht im vornhinein durch formelles Maß des Wiedererkennens festgelegt. Man kann dem Konventionalismus der Sprechakttheorie $^{26}$ kein freies Flottieren von Performanz entgegensetzen, denn eine schlichte Umkehrung bestätigt nur die Hierarchie. Auch das Neue bedarf eines Maßstabs für den Vergleich.

Man muss die herkömmliche Position um ihre eigene Achse drehen, indem man die Konvention vom Grund zum Ziel der Erklärung macht. Während für die herkömmliche Position die Regel nicht nur Voraussetzung sprachlicher Performanz ist, sondern geradezu deren Möglichkeitsbedingung, wird die Regel jetzt zum Effekt der Wiederholung. Bezogen auf das Verhältnis von Gesetz und Verfahren wäre bei der herkömmlichen Position das Verfahren nur legitim als Repetition des Gesetzes, das heißt als identische Wiederholung; für die Dekonstruktion dagegen wäre das Verfahren die Iteration des Gesetzes, welche nicht nur wiederholt, sondern auch verschiebt. Es geht also nicht um die Abschaffung einer der beiden Seiten, sondern um eine Akzentuierung. Die einseitige Überordnung der Struktur über den Vollzug ist damit aufgelöst. ${ }^{27}$ Genauso wie sich beim Filmen das Drehbuch noch verändern kann, kann sich auch der Sinn des Gesetzes bei seiner Neuinszenierung im Verfahren verschieben. Das Verfahren ist keine Maschine, welche von einer Regel regiert wird, sondern gleicht eher einem von selbst gesetzten Zwängen geleiteten Stochern, das nachträglich als einigermaßen regelhaft beschrieben werden kann.

\section{Worum gebt's?}

Die Abschaffung absoluten Wissens in Form der wörtlichen Bedeutung ist die erste Voraussetzung für die demokratische Rückbindung der Rechtsanwendung. Wenn es aber zu einem Text eine Vielzahl von Lesarten gibt, muss sich entscheiden lassen, welche die beste ist. Hier erscheint Augsbergs Kriterium der Vor-

24 Vgl. dazu Harald Wohlrapp, Der Begriff des Arguments, Würzburg 2008, S. $47 \mathrm{ff}$.

25 Augsberg (Fn. 1), S. 74.

26 Vgl. dazu Jacques Derrida, Limited Inc., Wien 2001, wo neben den Aufsätzen von Derrida immerhin eine Zusammenfassung der Position von Searle abgedruckt ist, S. $47 \mathrm{ff}$.

27 Hier wäre vor allem die Arbeit von Davidson zu würdigen, den Augsberg (Fn. 1, S. 96) allerdings vollkommen zu Unrecht als Intentionalisten einordnet. 
aussagbarkeit innerhalb des juristischen Wissens. Wenn wir diese Voraussagbarkeit anhand abstrakter Regelungen beurteilen könnten, dann wäre die Souveränität des Königs in ein sicheres Exil gerettet. Er könnte in der Regel überleben. Das demokratische Moment der Diskussion im Verfahren könnte ihn nicht zur Rechenschaft ziehen. Aber zum Schrecken aller Berater des Königs geht der Jakobiner Augsberg hier einen Schritt weiter. Die unvermeidlichen Spannungen im juristischen Wissen müssen über Argumentation im Verfahren abgearbeitet werden. Das Verfahren ist kein Nürnberger Trichter für dumme Bürger, sondern der Ort, an dem das Recht sich durch die Argumentation der Prozessbeteiligten verschiebt. Demokratie beschränkt sich eben nicht nur auf das Gesetzgebungsverfahren, sondern in der Rechtsanwendung muss der hegemonialen Instrumentalisierung des Rechts entgegengetreten werden. Der Richter kann nicht einfach durchentscheiden und der Logik des Baumes folgen, sondern er muss die vorgetragenen Argumente und die vorhergehenden Entscheidungen aufnehmen. Wenn er dies tut, liefert er ein Stück der Selbstentmachtung des Rechts. ${ }^{28}$ Die zweite Voraussetzung liegt also darin, dass wir sowohl das epistemische Wissen in Form von Präjudizien als auch das wegen der Inhomogenität der Präjudizien unverzichtbare thetische Wissen anhand der Argumentation im Verfahren überprüfen müssen. Um den Deleuze variierenden Augsberg zu wiederholen: Man muss dann sagen, dass die herkömmliche Auffassung im Irrtum ist, soweit sie die Bindungen der Gerichte auf der Ebene der Regelsemantik sieht. Vergessen werden dabei die tatsächlich funktionierenden pragmatischen Bindungen. Diese gilt es im Rahmen eines nicht nur legalistischen, sondern auch verfahrensbezogenen Rechtsstaatsverständnisses zu entfalten. Erst damit ist das demokratische Potenzial eines dekonstruktiven Ansatzes im Recht entfaltet.

Die Demokratie bleibt im Kommen. Das Buch von Augsberg ist dafür ein wichtiger Schritt. Das Gesetz wird unlesbar als gerundete Totalität. Es wird lesbar als Streit, der immer nur vorläufig entschieden werden darf. Er verknüpft nicht nur auf fruchtbare Weise das Recht mit der Literatur, sondern er genügt vor allem den damit gesetzten sprachlichen Maßstäben. Sein Text ist klar geschrieben und trotzdem herrlich komplex. Man sollte es als Stilfibel für junge Juristen verwenden und natürlich als Stachel im Fleisch alter Rechtstheoretiker. 